\title{
The Role of Directionally Selective Neurons in the Perception of Global Motion
}

\author{
Tatiana Pasternak, Joanne E. Albano, and Daniel M. Harvitt \\ Department of Neurobiology and Anatomy and Center for Visual Science, University of Rochester, Rochester, \\ New York 14627
}

Dynamic random dot targets consisting of many localized motion vectors have been used to study the pooling of local motion signals into a global motion percept (Williams and Sekuler, 1984). In such displays, the dots are displaced with a constant step size and the direction of motion for each dot is chosen at random from a specified distribution. When the distribution extends over $360 \mathrm{deg}$, the display consists only of local random motion of individual dots and no coherent motion is reported. However, when the distribution is less than 360 deg (biased), the stimulus appears to flow in a single direction. We examined the effects of reducing the number of directionally selective (DS) cortical neurons on this integration process. Normal cats and cats with severely reduced proportions of DS neurons were trained on 2 direction discrimination tasks.

The discrimination of opposite directions was examined while varying either the range of directions of local motion, or the proportion of dots moving with biased distribution. When all dots in the display were directionally biased, cats with reduced numbers of DS neurons performed the task as well as normal cats and humans (threshold range: 280-320 deg). However, when the proportion of biased dots decreased, these animals had severe deficits. Thus, in the absence of noise, even a very small number of DS neurons can perform spatial pooling of local directional signals, and support normal discrimination of opposite directions. However, a full complement of directional detectors appears necessary when the motion signal is masked by noise.

The discrimination of small differences in direction revealed far more severe deficits, even when all the dots in the display were directionally biased (no noise). Cats with few DS neurons could not discriminate differences $<\mathbf{4 0} \mathbf{~ d e g}$, whereas normal cats discriminated differences of 9-12 degs. This deficit was even more pronounced when the targets were noisy or had a large range of directions. Thus, small differences in direction cannot be discriminated by a reduced complement of DS neurons, even in the absence of

Received Feb. 23, 1990; revised May 2, 1990; accepted May 4, 1990.

This work was supported by grants from the National Eye Institute, EY06175 (T.P.), EY07344 (J.E.A.) and EY01319 (Core Grant to the Center for Visual Science). William Vaughn helped develop software for generating dynamic random dot patterns. We wish to thank Marc Dorfman, Robert Sikora, and Larry Ota for excellent technical assistance. We also thank Vince Ferrera, Mary Hayloe, Walter Makous, and William Merigan for helpful discussions and comments on the manuscript.

Correspondence should be addressed to Tatiana Pasternak, Center for Visual Science, University of Rochester, Rochester, NY 14627.

Copyright (C) 1990 Society for Neuroscience $0270-6474 / 90 / 093079-08 \$ 03.00 / 0$ noise. This loss of accuracy is most likely due to the reduced sensitivity of directional mechanisms in cats that show a loss of DS in a majority of cortical neurons.

Motion signals arise when a target changes its position in space in a specified period of time (for a review, see Nakayama, 1985). Such local motion signals are detected by directionally selective (DS) cortical neurons and give rise to the perception of motion (e.g., Newsome et al., 1986). When several local motion vectors are present in the target, the visual system combines them into a global percept of motion. Adelson and Movshon (1982) used gratings to examine such integration of local motion signals. They studied how 2 orthogonal drifting gratings were perceived as a plaid moving in a single direction. Subsequently, Movshon et al. (1985) have shown that there are neurons in extrastriate middle temporal (MT) cortical area of the monkey that appear to respond to the direction of motion of the plaid rather than that of the component gratings. Williams and Sekulcr (1984) used dynamic random dot targets consisting of many localized motion vectors to examine the integration of local vectors over space and time. They have shown that observers perceive motion in the general direction of the mean of the distribution of directions in the target. They also have shown that such stimulus parameters as dot density, the size of displacement, and the range of the distribution affect the percept of global motion. More recently, Watamaniuk et al. (1989) showed that the precision with which observers are able to discriminate global direction of motion deteriorates both at short stimulus durations and as the range of direction vectors present in the target is increased.

Although these psychophysical studies provided important information about the spatial and temporal integration of local motion signals, little is known about the neural processing involved in vector integration. Newsome and Britten (1989) recorded responses of DS MT neurons of monkeys to dynamic random dot targets in which some dots moved coherently, while other dots moved at random (random step size and direction) and compared these responses to psychophysical performance. They found that the sensitivity of single neurons to coherence was similar to psychophysical thresholds and concluded that the MT neurons may have the necessary properties to account for psychophysical performance. Surprisingly, lesions of this area resulted in mostly transient deficits in psychophysical thresholds (Newsome and Paré, 1988).

The present study also focused on neural mechanisms that underlie the integration of local motion signals. However, rather than making a lesion limited to a single cortical area, we per- 
formed a "functional" lesion in which directional selectivity was greatly reduced in cortical areas that normally carry directional information (Cynader and Chernenko, 1976; Pasternak et al., 1985; Spear et al., 1985). With this type of manipulation we were able to examine the role of DS cortical neurons in the perception of global motion. Previous studies of these animals have demonstrated the importance of such neurons for the discrimination of direction and specd of low contrast, moving gratings (Pasternak and Leinen, 1986; Pasternak, 1987). In the present study, we varied the strength of the motion signal by manipulating the proportion of dots with a biased distribution of directions, not by varying stimulus contrast. We found that when all the dots in the distribution were biased (no noise), pooling of local motion vectors into a global motion percept took place even when the number of DS neurons was abnormally low. Thus, in the absence of noise, the discrimination of opposite directions of motion was nearly normal. However, when the targets were noisy, larger numbers of DS neurons were needed to discriminate directions. On the other hand, discrimination of small differences in direction could not be accomplished without a normal complement of DS neurons even in the absence of directional noise.

\section{Materials and Methods}

\section{Subjects}

Cats. Five adult cats were used. Three of the cats $(84,810,812)$ had been reared in an environment illuminated at $8 \mathrm{~Hz}$ by a $3-\mu \mathrm{sec}$ stroboscopic flash. The cats were exposed to this illumination 12 hours each day and were otherwise in total darkness. They remained in this environment for the first 8 months of life. Thereafter, they were removed to a normal laboratory environment. The other cats $(140,147)$ were reared normally. At the time of testing, both normal and strobe-reared cats were at least 5 -years-old and had had extensive behavioral testing on visual tasks involving moving and stationary patterns. Psychophysical performance of the 3 strobe-reared cats on these tasks has been described elsewhere (Pasternak, 1986, 1987; Pasternak and Leinen, 1986).

Neurons in the striate cortex of strobe-reared cats show a normal incidence of orientation selectivity, temporal frequency response, and contrast sensitivity (Pasternak et al., 1985). On the other hand, DS of these neurons is nearly abolished; only about $5 \%$ of cells can be classified DS (compared to $64 \%$ DS neurons in normal cats). The only other receptive field abnormalities are slight reductions in binocularity and spatial resolution. Recordings from the lateral suprasylvian area in these cats also showed a profound reduction in DS, but other receptive field properties are intact (Spear et al., 1985). Psychophysical testing of these cats revealed only minor deficits in spatial resolution, nearly normal contrast sensitivity for detecting gratings flickering in counterphase, and only slightly reduced sensitivity for moving gratings (Pasternak and Leinen, 1986; Pasternak, 1986). On the other hand, these cats showed a 10 -fold loss in sensitivity for identifying the direction of moving gratings (Pasternak et al., 1985; Pasternak and Leinen, 1986), and severe deficits in the discrimination of speed and temporal frequency (Pasternak, 1987). It is important to note that strobe-reared cats used in the present study are littermates or had been reared together with animals used in physiological studies.

During testing, the cats were maintained at $80-85 \%$ of their normal body weight. Water was continually available in the home cage, and they received a daily supplement of Purina Chow.

Human observers. Four human observers were tested. One was a wellcorrected myope (TP); the other 3 required no optical correction $(\mathrm{DH}$, $\mathrm{MD}, \mathrm{VHF}$ ).

\section{Stimuli}

We used dynamic random dot targets first introduced by Williams and Sekuler (1984) and more recently used by Watamaniuk et al. (1989). The stimuli were generated on a PDP11/73 computer and displayed on a Tektronix 606 oscilloscope (P-31 phosphor). In such targets, the dots are displaced by a constant step size within a given temporal interval, and the direction of motion for each dot is randomly chosen from a specified uniform distribution of directions. The speed with which the dots are displaced is determined by a combination of the step size $(\Delta x)$ and temporal interval between steps $(\Delta t)$. The directions of dot displacement are randomly sampled every $1.8 \mathrm{deg}$ from a specified distribution. When the extent of the distribution of directions is $360 \mathrm{deg}$, the display consists only of local random motion of individual dots, and no coherent motion is reported. However, when the distribution is less than $360 \mathrm{deg}$, the dots appear to flow coherently in a single direction. The saliency of the stimulus was manipulated by varying the range of the direction distribution (direction range) and by varying the proportion of dots with biased direction distribution (\% motion signal) as shown in Figure 1.

The stimuli were placed $35 \mathrm{~cm}$ from the observer, behind 2 immediately adjacent 7-deg-dia circular openings in a white surround. Each display contained 50 dots, and the dot density in all experiments presented here was $0.94 \mathrm{dot} / \mathrm{deg}^{2}$, and mean luminance of the display was $0.1 \mathrm{~cd} / \mathrm{m}^{2}$. Each dot subtended $0.08 \mathrm{deg}$ dia and its luminance was set $3.5 \mathrm{log}$ units above detection threshold for human observers. Under these conditions there was no indication of streaks produced by moving dots.

\section{Procedure}

Cats. In a 2-alternative spatial forced-choicc paradigm, the cats viewed the 2 stimuli through 2 glass response panels and were rewarded for a nose-pressing response toward the correct stimulus. To make the viewing time more uniform, the stimuli were presented for $2 \mathrm{sec}$ before the cat's response was effective. The latency of each response was recorded. During each trial, the cats were presented either with 2 patterns that moved in opposite directions (rightward vs. leftward); 2 patterns, 1 with a direction distribution of $360 \mathrm{deg}$ (only random local motion), and the other with biased distribution of directions; or with patterns that differed in direction by $90 \mathrm{deg}$ or less (rightward vs. downward or oblique), depending upon the experiment. They were rewarded with pureed beef for choosing the dot pattern that moved rightward. A response towards any other direction resulted in a 10-sec tone and no food reward. The next trial began following a 5-sec intertrial interval.

Humans. The stimuli and the procedure used with human observers were very similar with the following exceptions: the dots were presented for a period of $1 \mathrm{sec}$, the intertrial period lasted $3 \mathrm{sec}$, and auditory feedback was used to indicate correct and incorrect responses. Observers used a chinrest and fixated a spot placed between the 2 targets.

\section{Experiment 1: discrimination of opposite directions of motion}

Initially, the cats were trained with targets in which all dots ( $100 \%$ signal) moved in a single direction (direction range, $0 \mathrm{deg}$ ). The stimuli always moved at $10 \mathrm{deg} / \mathrm{sec}$. This speed was achieved by setting the $\Delta x$ to 0.3 deg and the $\Delta t$ to $30 \mathrm{msec}$. After the cats reached criterion performance ( 3 consecutive 200 trial sessions $>80 \%$ correct), threshold measurements began. Two types of thresholds were measured: maximal direction range at scveral levels of motion signal $(100,50,25,12$, and $6 \%$, and the minimal motion signal required for discriminating opposite mean directions for several direction ranges $(1,90,180$, and $270 \mathrm{deg})$. A staircase procedure was used in which 3 consecutive correct responses resulted in an increase in the range of direction distribution (or decrease in \% motion signal), while each incorrect response resulted in the reverse. Thresholds were taken at $75 \%$ correct from the resulting psychometric function.

\section{Experiment 2: direction difference thresholds}

The cats were trained on this task after extensive training in Experiment 1. For this task, a speed of $30 \mathrm{deg} / \mathrm{sec}(\Delta x, 0.9 \mathrm{dcg} ; \Delta t, 30 \mathrm{msec})$ was used, since previous experiments have shown that direction difference thresholds in cats are optimal at speeds greater than $10 \mathrm{deg} / \mathrm{sec}$ (Pasternak and Merigan, 1984). Although direction discrimination with dynamic random dots may depend upon step size (Williams and Sekuler, 1984), we have found that for a dot density of $0.94 \mathrm{dots} / \mathrm{deg}^{2}$ the optimal step size for cats extends to at least $1 \mathrm{deg}$ (T. Pasternak, unpublished observations). The cats were first presented with rightward and downward moving targets and usually required only 1 session to reach 85 $90 \%$ correct performance. Direction difference thresholds were measured for several levels of motion signal $(100,50,25$, and $12 \%)$ and for several direction ranges $(1,90,180$, and $270 \mathrm{deg})$. Since the directions of dot displacement were sampled randomly every $1.8 \mathrm{deg}$, direction thresholds smaller than 2 deg could not be measured. Under the staircase 


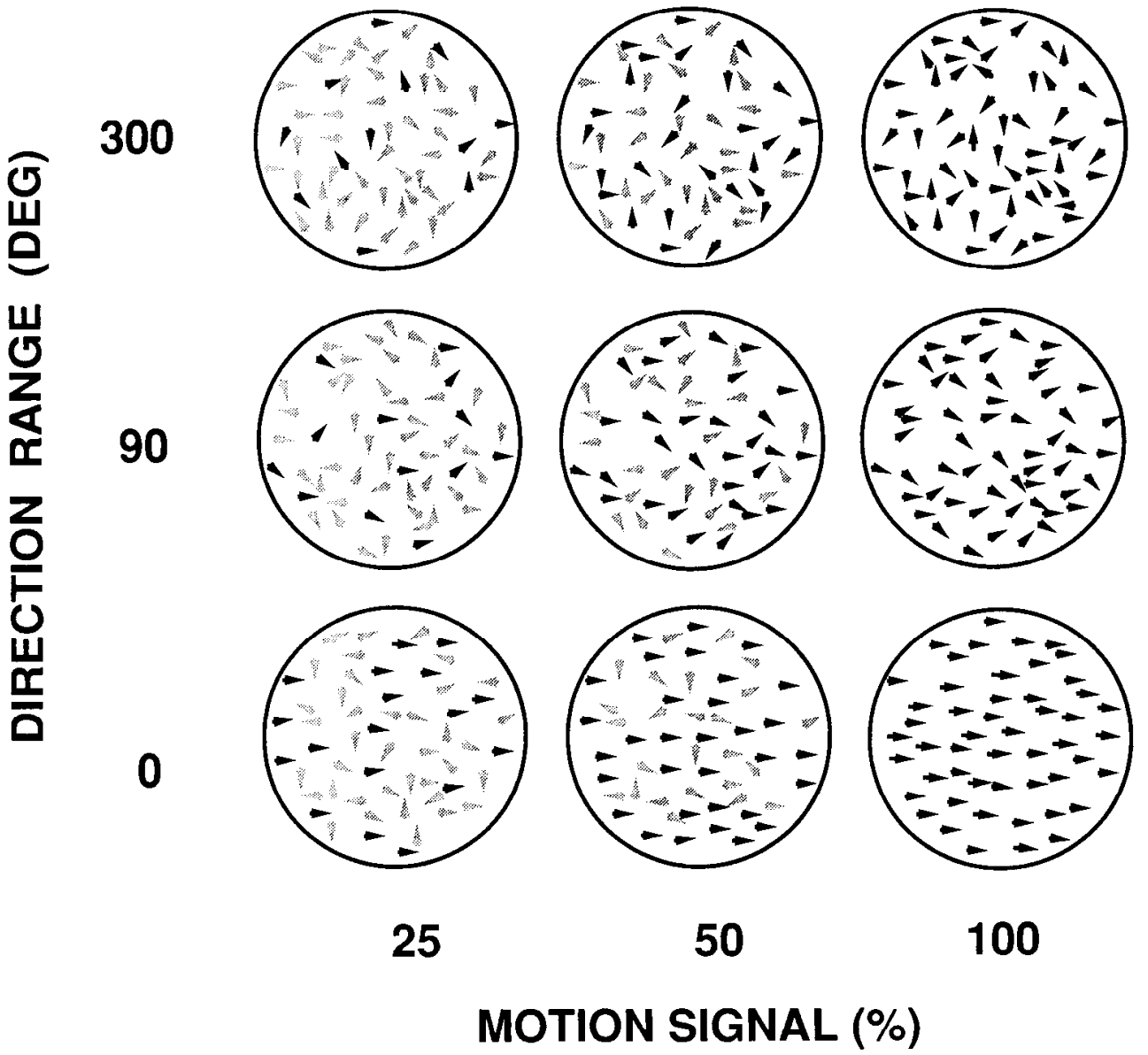

Figure 1. Schematic illustration of targets used in the study. Each arrow represents the direction of motion of an individual dot. Each dot is displaced with a constant step size (see Materials and Methods). The variation in direction range is shown along the vertical axis, while variation in the proportion of dots with the biased direction distribution (\% motion signal) is shown along the horizontal axis. When the range is $360 \mathrm{deg}$, the distribution of directions is unbiased and the observer perceives only local displacements of individual dots; no global motion is reported. As the direction range is decreased to about 300 deg, observers report global motion and are able to discriminate opposite directions. When the range is set to 0 (bottom row) the dots in the distribution are displaced in the same direction. When all the dots in the distribution are directionally biased (right column), motion signal is $100 \%$. The strength of motion signal was manipulated by varying the proportion of directionally biased dots. Light arrows (middle and left columns) show the proportion of dots with an unbiased distribution of directions (range, $360 \mathrm{deg}$ ). The middle column shows the display in which $50 \%$ of dots is directionally biased, while the remaining $50 \%$ has the direction distribution of $360 \mathrm{deg}$. The left column shows the display where only $25 \%$ of the dots is directionally biased. procedure, 3 consecutive correct responses resulted in a decrease in direction difference, while each incorrect response resulted in an increase in difference between the standard (rightward) and the comparison direction. As in Experiment 1 , the threshold was the stimulus value that corresponded to $75 \%$ correct on the psychometric function.

\section{Results}

Experiment 1: discrimination of opposite directions of motion In this experiment we asked observers to discriminate between 2 targets: 1 with a biased direction distribution centered on rightward, the other with direction distribution centered on leftward. To human observers, these displays appeared to move in opposite directions. We examined the discriminability of directions by varying the number of directions present in the display, and the percentage of dots in the display that had a biased distribution of directions. This study was aimed at determining whether discriminability of opposite directions is degraded by a severe reduction in the number of DS cortical neurons.

We first determined the greatest direction range at which normal observers could reliability discriminate between rightward and leftward motion. Direction range thresholds for the 2 normal observers could reliably discriminate between rightward function of percent motion signal (i.e., proportion of directionally biased dots). The overall performance of the 2 species was similar, although the functions for the cat and human deviated somewhat at low signal levels (12 and 6\%). When all the dots in the distribution were biased ( $100 \%$ motion signal), normal cats and humans were able to extract global direction even up to direction ranges over $300 \mathrm{deg}$. This performance was seriously degraded only when the proportion of dots with biased distribution of directions was reduced to $12 \%$ in cats and below $6 \%$ in humans.

Figure 3 shows a comparison of mean range thresholds in 2 normal cats (open circles) and in cats with a reduced proportion of DS neurons (filled circles) for discriminating opposite directions of motion. Individual mean thresholds for the 2 normal cats were shown in Figure 2. Individual cats with reduced DS performed quite similarly as shown by error bars. Individual SEMs ranged 5-20 deg. Cats with reduced DS performed nearly as well as normal cats when $100 \%$ of dots were biased. However, their performance was seriously degraded when the proportion of directionally biased dots was decreased to $50 \%$. As the motion signal was reduced below $25 \%$, they were totally unable to discriminate opposite directions.

We also wanted to see whether the deficits of cats with reduced DS were still present if the animals were not required to identify target direction, but were asked only to discriminate between 2 targets, 1 with an unbiased distribution (noise; range $=360 \mathrm{deg}$ ) and the other with a biased distribution of directions. The results, plotted as squares, show even greater deficits in performance, suggesting that even when the animals are not required to identify target direction, DS mechanisms were necessary for the analysis of dynamic random dot targets.

The thresholds shown above were obtained by manipulating the range of directions in the target within 1 session. One consequence of an increase in the direction range is a reduction in the "net" displacement of dots over time, i.e., the target appears 
Figure 2. Direction range thresholds for discriminating opposite directions of motion. Data for, left, 2 human observers (TP, circles; DH, triangles) and, right, 2 normal cats $(140$, circles; 147 , triangles). The thresholds for the $2 \mathrm{hu}-$ man observers, shown by filled symtion signal for dots with direction range set to $1 \mathrm{deg}$ (see text and Figure 5). Error bars are SEM. bols, were measured by varying \% mo-

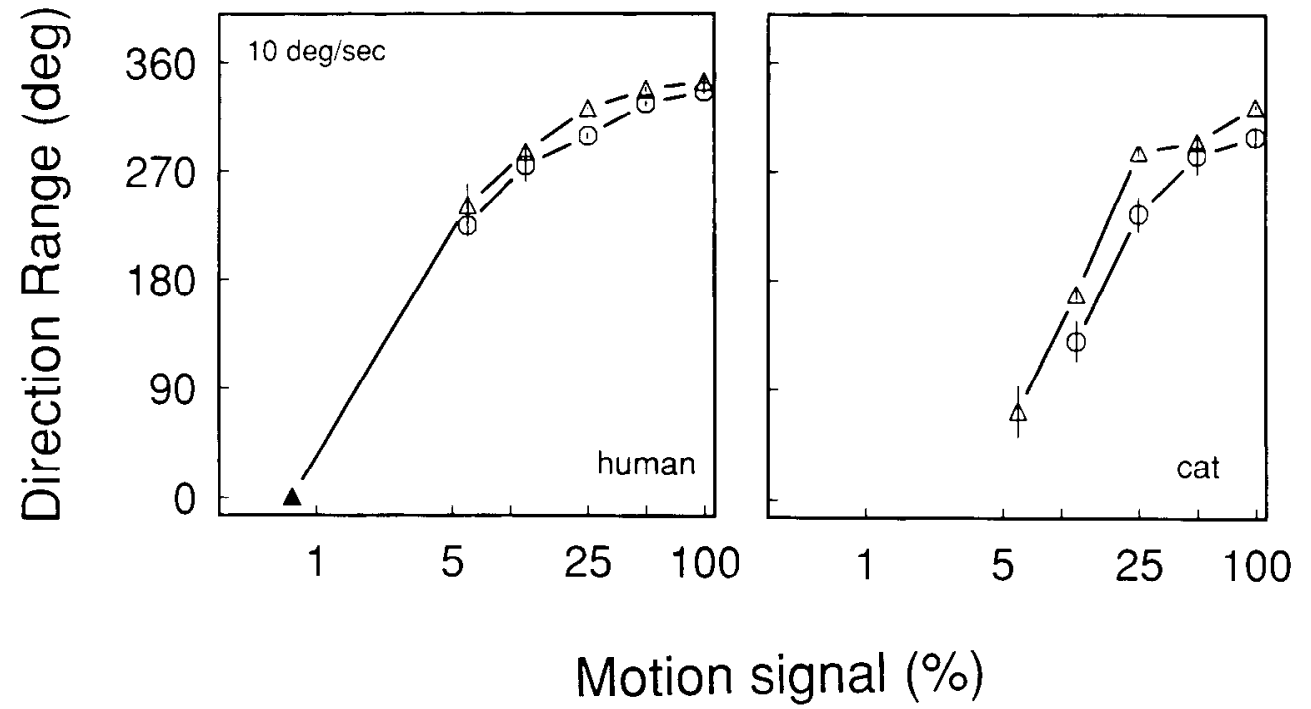

to drift over shorter distances and the apparent speed is reduced. We were concerned that the range thresholds shown in Figures 2 and 3 might be limited by the global distance traveled by the dots, rather than by the range of directions per se. Thus, we assessed direction discrimination by varying \% motion signal while keeping the direction range constant. The results of this measurement for 1 normal cat and 2 human observers are shown in Figure 4. The threshold motion signal (proportion of dots with biased distribution of directions) required to discriminate opposite directions is plotted. The performance of the 2 species was quite similar, although human observers discriminated directions at slightly lower levels of motion signal.

The same type of threshold is plotted in Figure 5 for the 3 cats with reduced DS and 2 normal cats. The performance of

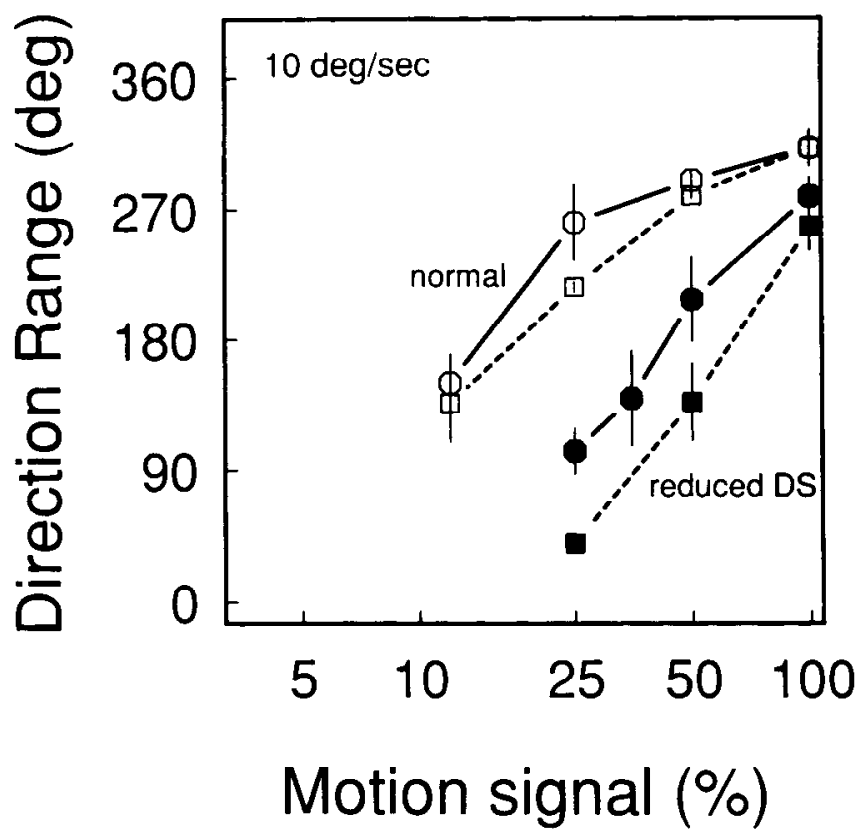

Figure 3. Direction range thresholds for discriminating opposite directions of motion (circles and solid lines) and for discriminating between directionally biased and unbiased (range $=360 \mathrm{deg}$ ) targets (squares and broken lines). Mean thresholds for 2 normal cats (open symbols) and 3 cats with reduced DS (filled symbols). Error bars are SEM. the 2 normal cats was not identical, particularly for broad direction ranges, with thresholds for cat 140 being substantially higher. This difference is reflected in large error bars for ranges of 180 and $270 \mathrm{deg}$. Individual thresholds for the deprived cats were quite similar with SEMs not exceeding 6\%. While normal cats were able to perform the direction discrimination at a range of $1 \mathrm{deg}$ when less than $10 \%$ of the dots were directionally biased (i.e., contributed to the motion signal), cats with reduced DS required about $40 \%$ of the dots to be directionally biased. A difference between the 2 groups of animals was found for all direction ranges. These thresholds are consistent with the range thresholds measured as a function of motion signal, shown in Figure 3.

This result suggests that the range thresholds shown in Figures 2 and 3 were most likely determined by the range of directions in the stimulus rather than by the apparent overall displacement of the target. It also provides another measure of the deficit in direction discrimination shown by cats with reduced DS by showing that these animals require much more signal (or less noise) for reliable discrimination of opposite directions of motion.

\section{Experiment 2: direction difference thresholds}

Although Experiment 1 provided information about some determinants of the integration of local motion vectors into a global unidirectional percept, it did not tell us about the precision with which cats with severely reduced DS could extract global direction from local vectors of the targets. Therefore, in the next experiment we asked observers to discriminate small differences in direction of 2 targets having somewhat different means of direction distributions, 1 with the distribution centered on rightward (standard) motion and the other centered on oblique down to the right (comparison). We measured the dependence of direction difference thresholds upon the number of directions in the distribution and upon the strength of the motion signal (proportion of dots with biased distribution of directions).

Thresholds at various levels of motion signal and as a function of direction range are shown for 2 normal cats and 2 human observers in Figures 6 and 7. As was the case for discrimination of opposite directions, human performance was quite similar to that of the normal cat, although somewhat more accurate. Under optimal stimulus conditions ( $100 \%$ signal, range $=0 \mathrm{deg})$, 


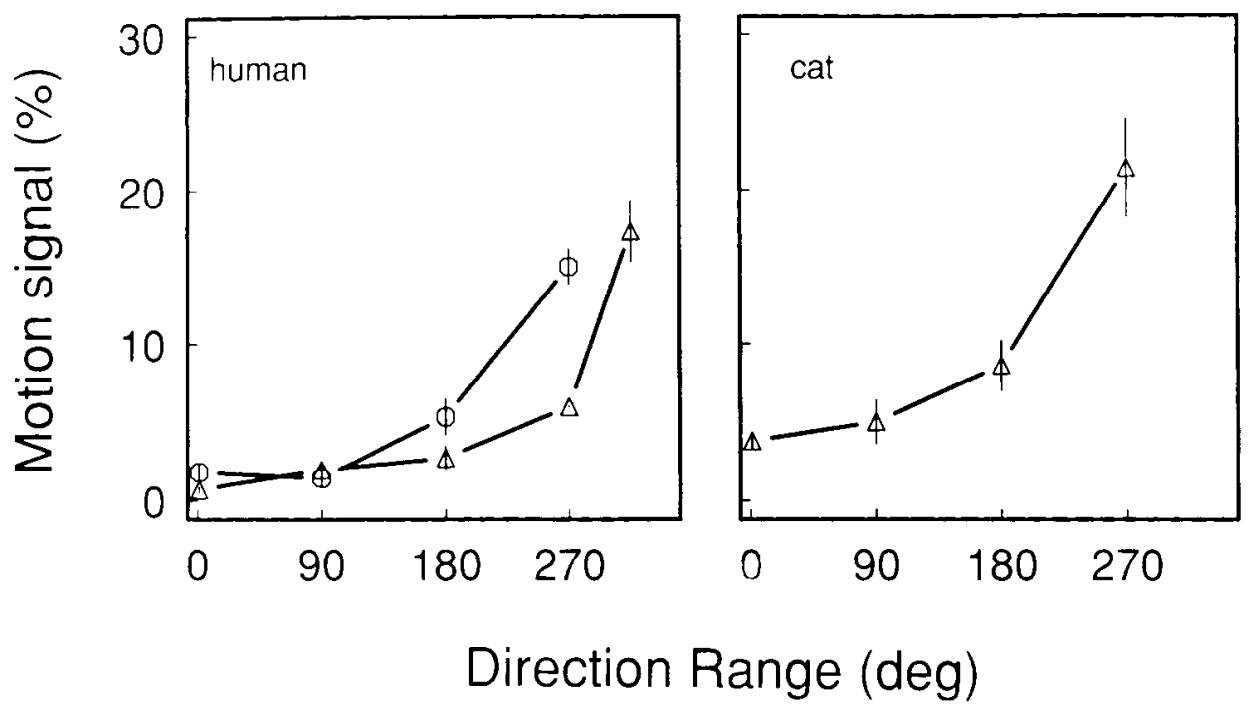

Figure 4. Proportion of dots with biased distribution of direction (\% motion signal) required for discriminating opposite directions of motion. Data for, left, 2 human observers (TP, triangles; $\mathrm{MD}$, circles) and, right, 1 normal cat (147) are plotted over a range of dircctions. Error bars are SEM. the cats discriminated approximately 9-12 deg differences in direction, while human performance was substantially more accurate (thresholds were $<2 \mathrm{deg}$ and could not be measured in our system; thresholds of about $2 \mathrm{dcg}$ wcre reported by Watamaniuk et al., 1989; and by De Bruyn and Orban, 1988). These direction thresholds strongly depended upon the presence of directional noise. As the motion signal was reduced, the accuracy of discrimination dropped drastically, and with only $12 \%$ of dots contributing to the motion signal, the cats could not discriminate differences $40 \mathrm{deg}$. Human performance remained somewhat more accurate under all stimulus conditions, although for larger direction ranges or lower signal strength, the performance of cats deviated only slightly from that of humans and appeared to show the same dependencies on stimulus manipulations.

Mean discrimination thresholds for the 3 cats with reduced DS are compared to those of 2 normal cats in Figure 8, $A$ and $B$. The performance of individual cats is not shown since their thresholds were quite similar, as shown by error bars. The SEM for individual cats did not cxcced $5 \mathrm{dcg}$. The performance of cats with reduced DS was seriously degraded even in the absence of noise ( $100 \%$ signal) or when the targets contained only a single direction (range $=0 \mathrm{deg}$ ). When the strength of the motion signal was reduced to $25 \%$, the direction threshold of the impaired cats rose to about $80 \mathrm{deg}$. When the same functions were measured with a broader distribution of directions (180 deg), the same pattern of results was found (not shown here), although the accuracy of discrimination was even lower than that for the range of $0 \mathrm{deg}$. While in normal cats a change in range from 0 deg to 270 deg reduced accuracy by a factor of 2 , this effect of range was not detectable in cats with reduced DS. The accuracy of their performance did not seem to be affected at all by an increase in the number of directions in the stimulus, except at the largest direction range $(270 \mathrm{deg})$, where the cats were unable to perform the task.

\section{Discussion}

The present study demonstrates that animals with reduced DS in the majority of their cortical neurons show less severe deficits when required to discriminate opposite directions than when asked to make more precise judgments about target direction. Deficits in the discrimination of opposite directions of motion were apparent only when the targets contained a high proportion of dots with a random distribution of directions (noise). In the absence of noise, these cats performed at a level only slightly below that of normal cats and humans. On the other hand, discrimination of small differences in direction was severely affected by the loss of DS, even when the targets contained no noise. Thus, while the integration of local vectors into a global motion percept can be accomplished by a very limited number of DS neurons, the discrimination of small differences in direction requires a normal complement of DS neurons.

\section{Discrimination of opposite directions}

Normal performance of cats and humans

The data show a remarkable ability of the visual system to extract global direction of dot patterns containing multiple di-

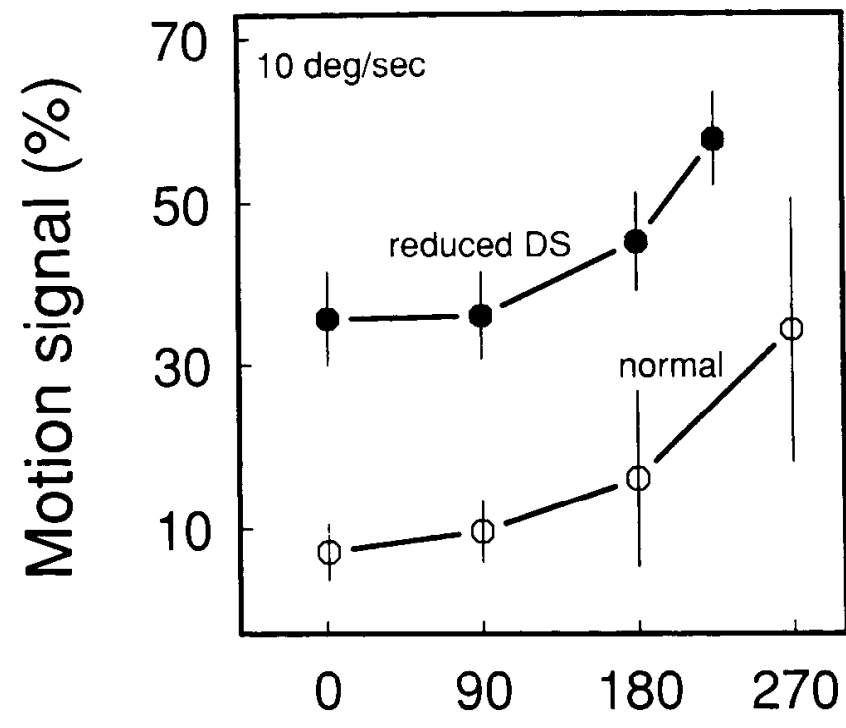

\section{Direction Range (deg)}

Figure 5. Proportion of dots with biased distribution of directions ( $\%$ motion signal) required for discriminating opposite directions of motion. Mean thresholds for 2 normal cats (open symbols) and 3 cats with reduced DS (filled symbols). Error bars are SEM. 
Figure 6. Dircction difference thresholds as a function of the proportion of dots with biased distribution of directions (\% motion signal). Left, 2 human observers (TP, circles; VHF, iriangles) and, right, 2 normal cats $(140$, circles; 147 , triangles).

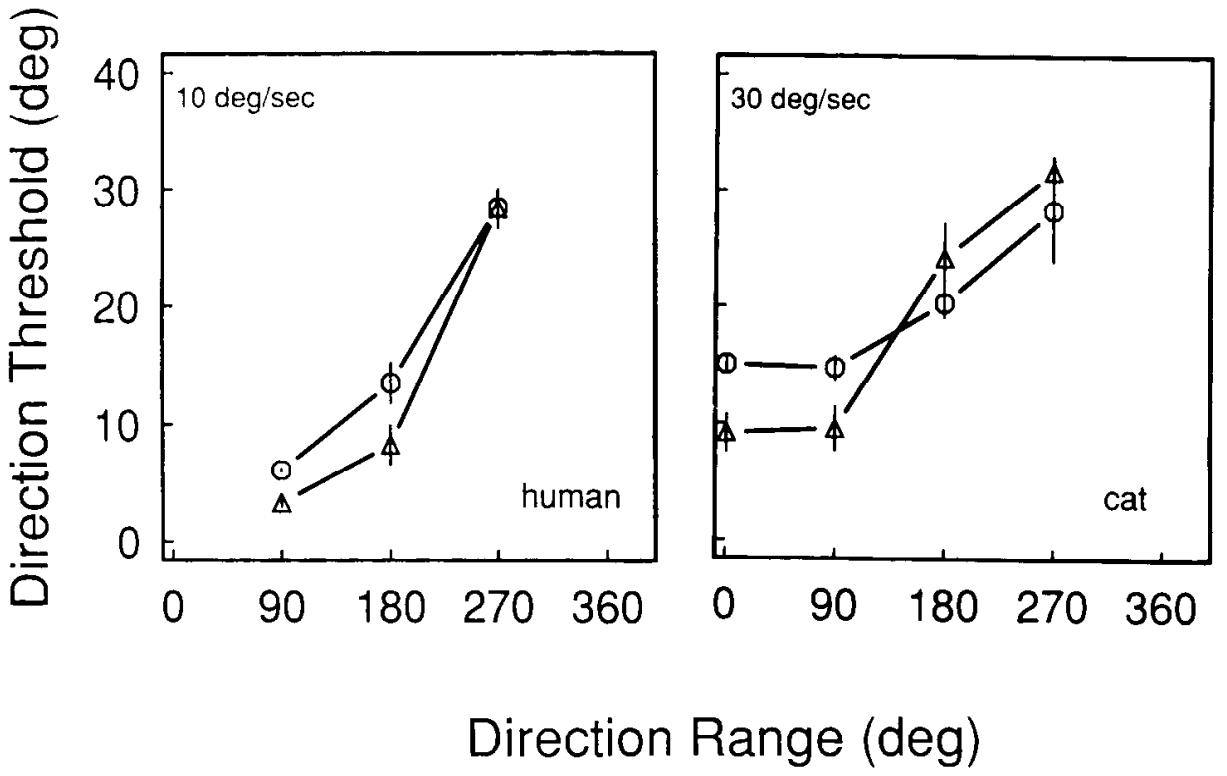

rection vectors. Adding noise to the target (by increasing the proportion of dots with distribution of $360 \mathrm{deg}$ ) degrades the performance, and limits detection to stimuli with a narrower distribution of directions. The relationship between the range threshold and the proportion of biased dots was similar in cats and humans, although cats required a somewhat larger proportion of biased dots (\% motion signal) than humans to perform the same task. Of course, comparison of absolute threshold values is problematic since the conditions chosen for 1 species as optimal may not be optimal for the other. Among factors that are likely to influence direction thresholds are target speed (Pasternak and Merigan, 1984), and dot density (Williams and Sekuler, 1984; T. Pasternak, unpublished observations), with cats showing the best range thresholds at speeds above $20 \mathrm{deg} / \mathrm{sec}$ (T. Pasternak, unpublished observations). For the study of the discrimination of opposite directions we chose identical dot density $\left(0.94 \mathrm{dots} / \mathrm{deg}^{2}\right)$ and speeds $(10 \mathrm{deg} / \mathrm{sec})$ for the 2 species. While the dot density appears to be within the optimal range for both species (T. Pasternak, unpublished observations), the speed is somewhat below optimal for cats. This may account for the differences in thresholds between the 2 species.

A similar relationship between the detectability of unidirectional motion and the range of the direction distribution was previously reported by Williams and Sekuler (1984), who first introduced the stimuli used in the present study. Their observers rarely reported seeing unidirectional motion when the range of directions in the targets approached $270 \mathrm{deg}$, a somewhat narrower range than that measured for our human and cat observers. This difference is most likely due to the lower dot density used in the present study (see Williams and Sekuler, 1984; T. Pasternak, unpublished observations). The use of a spatial forcedchoice procedure in the present study may have contributed to better thresholds, since our observers had an opportunity to view simultaneously the targets to be discriminated. Williams and Sekuler also manipulated the proportion of dots in the target that were biascd, and as the present study, found that the ob-
Figure 7. Direction difference thresholds as a function of direction range. Left, 2 human observers (TP, triangles; $\mathrm{DH}$, circles) and, right, 2 normal cats (140, circles; 147 , triangles). Error bars are SEM.
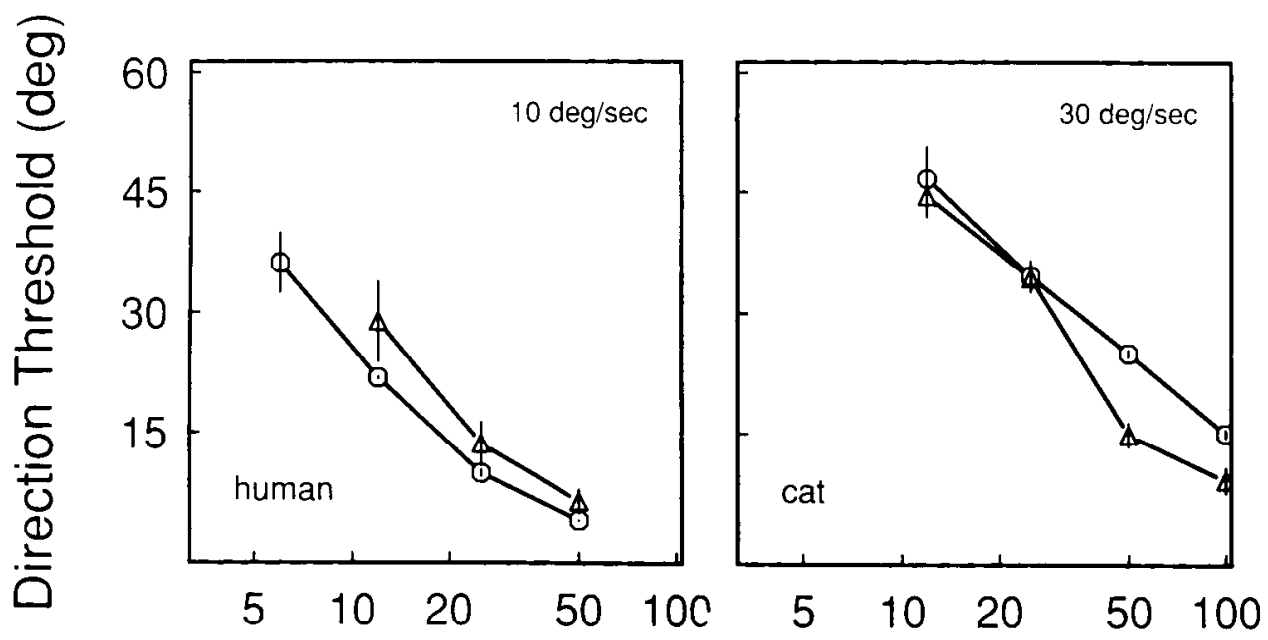

Motion signal (\%) 


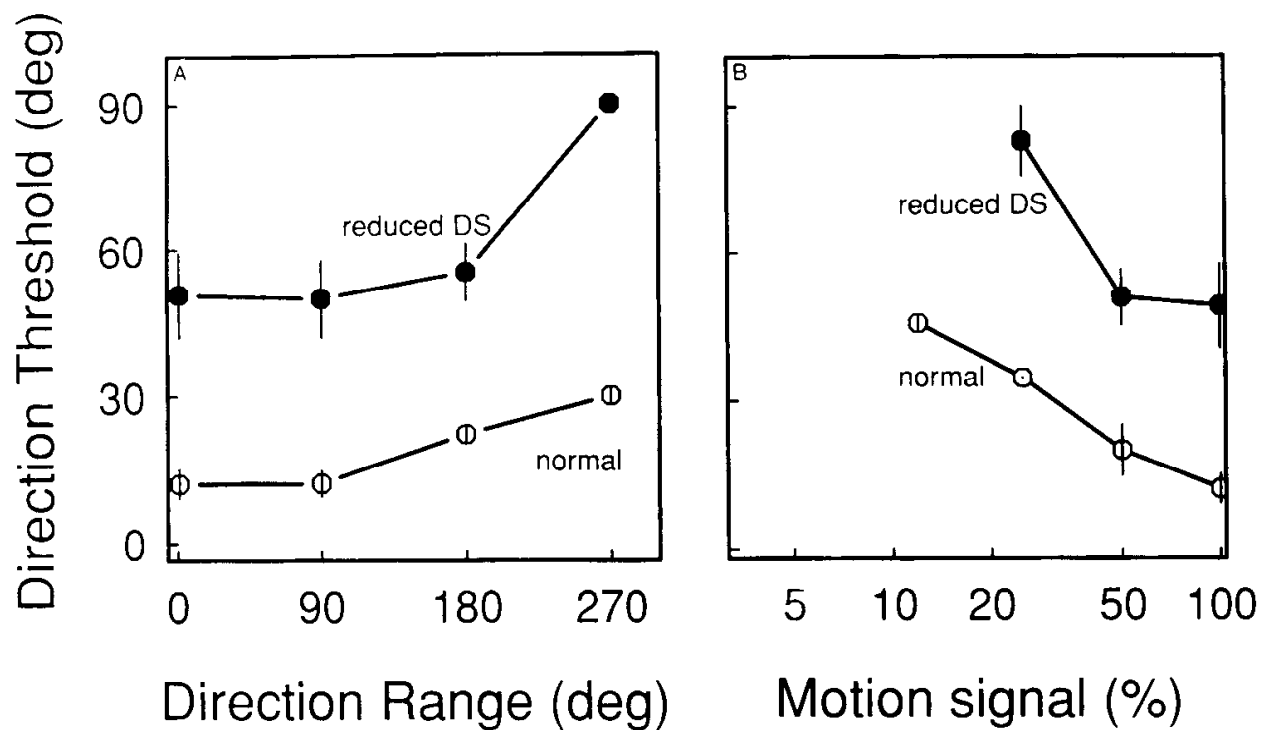

Figure 8. Mean direction difference thresholds as a function of direction range $(A)$ and as a function of $\%$ motion signal $(B)$ for 2 normal cats (open symbols) and 3 cats with reduced DS (filled symbols). Error bars are SEM. servers were unable to identify unidirectional motion when motion signal dropped below $10 \%$.

The targets used here are in some ways similar to those used by Newsome and Pare (1988) in their investigation of the effects of MT lesions on motion perception, and later by Newsome and Britten (1989) and Newsome et al. (1989) in the study of psychophysical and single unit responses to dynamic random dot patterns. In their display, the masking dots were displaced in random directions and with random step size, and the detectability of motion was manipulated by correlating both the direction and speed of individual dots. The motion of correlated dots was always in a single direction. In contrast, in our display the step size was constant, and the detectability of motion was manipulated by varying the number of directions in the target or the proportion of dots with a random distribution of directions. Despite this difference, under some stimulus conditions the displays used in the 2 studies are quite similar. Thus, the stimuli used to measure correlation thresholds are similar to those we used to measure \% motion signal threshold when all biased dots moved in a single direction (range $=0 \mathrm{deg}$ ). Indeed, the performance of human $(1-2 \%)$ and cat $(4-10 \%)$ observers in the present study overlaps with that of monkeys $(2-5 \%)$ in Newsome's study (Newsome and Paré, 1988).

\section{Cats with reduced cortical directional selectivity}

The most striking finding of the present study is that in the absence of noise, a severe loss of directional selectivity had very little effect on the ability to extract global direction from targets containing a broad range of local directional vectors. This result provides some insights into the way the visual system performs the integration of motion vectors required by the task. By removing directions near the mean from the distribution of directions, Williams and Sekuler (1984) provided evidence that the resulting global percept is a product of pooling of detectors tuned to the mean direction of the distribution. Our result shows that even a very small number of directional detectors is sufficient to perform such pooling. The most likely explanation for the poor performance of these animals with noisy targets is the extremely low sensitivity of their directional mechanisms associated with the reduced number of DS neurons (Pasternak and Leinen, 1986). Such insensitive mechanisms require robust signals and the degradation of signals by the presence of noise is likely to reduce their performance.

Newsome and Paré (1988) used dynamic random dot targets (see above) to examine the role of cortical area MT in motion processing. This area has a very high proportion of DS neurons (e.g., Movshon et al., 1985) that respond reliably to such targets (Newsome et al., 1989). They found that lesions of this area resulted in a 5-10-fold threshold clcvation, a loss similar to that found in cats with reduced DS in this study. However, this deficit decreased with time, and after 9 days the performance returned to normal levels. The authors proposed that this recovery might be a reflection of the activity of other pathways capable of carrying motion information in parallel with MT. Results of this study provide important insights into the mechanism of behavioral recovery following lesions limited to a single cortical area (also see Newsome et al., 1985). They demonstrate that when the ability of all major pathways normally carrying DS is severely limited, no behavioral recovery is possible. This result, in conjunction with the recovery of function observed after extrastriate lesions in monkeys and cats (Newsome et al., 1985; Newsome and Paré, 1988; Pasternak et al., 1989; T. Pasternak, unpublished observations), suggests that a single cortical area is unlikely to mediate discrimination of stimulus direction, even if this area has a large number of DS neurons.

\section{Discrimination of small differences in direction}

\section{Normal performance of cats and humans}

For this task, we optimized stimulus conditions by choosing stimulus speeds previously found to be optimal for direction discrimination for the 2 species (Pasternak and Merigan, 1984). Under these conditions, direction thresholds strongly depended upon the range of directions in the stimulus; as the range of directions increased, the accuracy of performance decreased. This relationship was found in both species, although the accuracy of discrimination in cats was lower than that of humans, particularly for targets with the narrow range of directions. A similar relationship between the direction range and direction discrimination thresholds has previously been reported for humans for narrower direction ranges (Watamaniuk et al., 1989). 
The discrimination thresholds were also very sensitive to the presence of directional noise in the target, and again human observers were somewhat more accurate than cats. Surprisingly, the difference in performance was less pronounced when the targets contained a larger range of directions or were noisy. One factor that could explain the greater similarity between the 2 species under these conditions may be the difference in viewing time. While cats could have viewed the targets for up to 2-3 sec, human observers were allowed to inspect the targets for only $1 \mathrm{sec}$. Although direction discrimination for targets containing a small range of directions asymptotes around $500 \mathrm{msec}$, asymptotic performance for targets with broad range of directions is not reached even at $1000 \mathrm{msec}$ (Watamaniuk et al., 1989). Thus, the shorter viewing time could have reduced the accuracy of performance of human observers for targets containing a broad range of directions.

\section{The role of cortical directional selectivity}

The present data showed that the precision with which direction differences are discriminated is degraded when the number of DS neurons in the visual cortex is abnormally low. This result is consistent with predictions of the line-element model previously used to account for spatial frequency and orientation discrimination thresholds (Wilson and Gelb, 1984; Wilson and Regan, 1984), and more recently to model direction discriminations (Watamaniuk et al., 1989). According to this model, discrimination of small differences in direction is based on a computation of differences in the response of local detectors (e.g., DS neurons or mechanisms) and the subsequent pooling of these differences across directions. The sensitivity of these local detectors and the slope of their tuning functions are the major determinants of the accuracy of discrimination (Wilson and Gelh, 1984). Thus, one would expect degradation in the discrimination performance if the sensitivity of local directional mechanisms were reduced and/or if the slope of the tuning functions were shallow. Indeed, the sensitivity of directional mechanisms in cats with reduced DS of cortical neurons is severely reduced (Pasternak and Leinen, 1986). Thus, loss of direction discrimination accuracy in cats with reduced DS is consistent with predictions of the line-element model. This argues in favor of the mechanism proposed by the model that direction discrimination is based on the differential response of local mechanisms tuned to direction.

Deficits in discriminating high contrast targets in cats with reduced DS are limited to the domain of motion perception [i.e., direction (the present study) and speed (Pasternak, 1987)]. Ilowever, asked to discriminate differences in the orientation of stationary, high-contrast gratings, such cats perform similarly to normal cats (Pasternak and Leinen, 1986). This dissociation of responses to stimulus orientation and direction parallels the results of single-unit recordings from striate cortex of these cats (Pasternak et al., 1985) and cats reared under identical conditions (Cynader and Chernenko, 1976). Such cats show a 90\% reduction in the number of DS neurons but a normal number of orientation selective neurons and a normal width of orientation tuning. Thus, mechanisms that underlie the discrimination of direction and orientation are likely to be distinct.

\section{Concluding remarks}

Comparison of human and cat performance on these tasks revealed important similarities in the pooling of local vectors into a global motion percept. Despite differences in absolute threshold values, the dependence of the discrimination functions on various stimulus manipulations was remarkably similar. The similarity of this motion integration process in such diverse species as cat and human suggests that it is likely to be a general property of the mammalian visual system. The severe deficits observed in cats with reduced DS in visual cortical neurons show that DS ncurons play a critical rolc in this process.

\section{References}

Adelson EH, Movshon JA (1982) Phenomenal coherence of moving gratings. Nature Lond 300:523-525.

Cynader M, Chernenko G (1976) Abolition of directional selectivity in the visual cortex of the cat. Science 193:504-505.

De Bruyn B, Orban GA (1988) Human velocity and direction discrimination measured with random dot patterns. Vision Res $28: 1323-$ 1335 .

Movshon JA, Adelson EH, Gizzi MS, Newsome WT (1985) The analysis of moving patterns. In: Pattern recognition mechanisms (Chagas C, Gattass R, Gross C, eds), pp 117-151. Berlin: Springer.

Nakayama K (1985) Biological image processing: a review. Vision Res 25:625-660.

Newsome WT, Britten K (1989) Neuronal correlates of a perceptual decision. Nature 341:52-54.

Newsome WT, Paré EB (1988) A selective impairment of motion perception following lesions of the middle temporal visual area (MT). J Ncurosci 8: 2201-2211.

Newsome WT, Wurtz RH, Dursteller MR, Mikami A (1985) Deficits in visual motion processing following ibotenic acid lesions in the middle temporal visual area of the macaque monkey. J Neurosci 5 : 825-840.

Newsome WT, Mikami A, Wurtz RH (1986) Motion selectivity in macaque visual cortex. III. Psychophysics and physiology of apparent motion. J Neurophysiol 55:1340-1351.

Newsome WT, Britten KH, Movshon JA, Shadlen M (1989) Single neurons and the perception of visual motion. In: Neural mechanisms of visual perception (Lam DK, Gilbert C, eds), pp 171-198. The Woodlands, TX: Portfolio.

Pasternak T (1986) The role of directional selectivity in detection of motion and flicker. Vision Res 26:1187-1194.

Pasternak T (1987) Discrimination of differences in speed and flicker rate depends on directionally selective mechanisms. Vision Res 27: 1881-1890.

Pasternak T, Leinen L (1986) Pattern and motion vision in cats with selective loss of cortical directional selectivity. J Neurosci 6:938-945.

Pasternak T, Merigan WH (1984) Effects of stimulus speed on direction discrimination. Vision Res 24:1349-1355.

Pasternak T, Schumer RA, Gizzi MS, Movshon JA (1985) Abolition of cortical directional selectivity affects visual behavior in cats. Expl Brain Res 61:214-217.

Pasternak T, Horn KM, Maunsell JHR (1989) Deficits in speed discrimination following lesions of the lateral suprasylvian cortex in the cat. Visual Neurosci 3:365-375.

Spear PD, Tong L, McCall MA, Pasternak T (1985) Developmentally induced loss of direction sclective ncurons in cat's lateral suprasylvian cortex. Developmental Brain Res 20:281-285.

Watamaniuk SNJ, Sekuler R, Williams DW (1989) Direction perception in complex dynamic displays: the integration of direction information. Vision Res 29:47-59.

Williams DW, Sekuler R (1984) Coherent global motion percepts from stochastic local motions. Vision Res 24:55-62.

Wilson HR, Gelb DJ (1984) Modified line element theory for spatial frequency and width discrimination. J Opt Soc Am A 1:124-131.

Wilson HR, Regan D (1984) Spatial frequency adaptation and grating discrimination: predictions of a line-element model. J Opt Soc Am A 1:1091-1096. 